\section{SATELLITE SYMPOSIUMS ABSTRACTS}

\section{ROCHE SATELLITE SYMPOSIUM}

\section{MANAGEMENT OF A COAGULATION LABORATORY IN COVID-19 PANDEMIC}

Oguzhan Zengi

Basaksehir Cam and Sakura City Hospital Center Laboratory, Istanbul

The COVID-19 virus causes coagulation-related disorders in affected patients in a way that has not been seen before. Coagulation laboratories, which are of particular importance due to their pre-analytical, analytical, and post-analytical vulnerabilities, executed high test numbers during the pandemic period and became even more vulnerable to pre-analytical and post-analytical errors. Scientific requirements in the management of the coagulation laboratory under pandemic conditions will be discussed in this symposium. The experiences of Istanbul Basaksehir Cam and Sakura City Hospital Center Laboratory, in which Roche Diagnostics Preanalytical solution systems and T711 coagulation analyzer are installed, will be shared

\section{BECTON DICKINSON SATELLITE SYMPOSIUM BD COVID-19 SOLUTIONS AND POC ANTIGEN TESTING}

Ipek Cinaroglu, Bahityar Can, Begum Yildiz Becton Dickenson Life Sciences-Integrated Diagnostic Solutions, Istanbul

OBJECTIVES: COVID-19 assays categorized as molecular assays used for diagnosis, rapid antigen tests (RAT) used for diagnosis and screening, and antibody assays determining the previous infection.

RATs are included in the guidelines of WHO, CDC, and ECDC. POC tests increase the possibility of testing and shorten the time to result. The clinical performance of RATs depends on the conditions in which they are used.1,2,3

MATERIALS AND METHODS: The accuracy increased when tested in the early stages of infection with SARS-CoV-2, when viral load and the prevalence is high2. RATs can be used for screening in high risk environments; quickly identify patients with active infections. Thus, can prevent contamination.1,2,3 Where rapid results are required, RATs are valuable to provide instant results even sensitivity lower than RT-PCR.

RESULTS: The parameters affecting accuracy in RATs can be summarized as correct sampling, prevalence, presence of symptoms, sampling period, and accuracy and sensitivity of the assay.

CONCLUSION: BD Veritor TM Plus RATs stands out with easy sample processing capability, 15 minutes resulting-time and reliable performance. Studies show $\% 84$ Positive and \%100 Negative Agreement, and these values are higher with the increased prevalence. 4

It is important to evaluate the immune system for the follow-up of COVID- 19 . Studies have shown that individuals with COVID-19 have a decrease in T cell subgroups CD4 and/or CD8+T cell counts with increasing disease severity. The correct $\mathrm{T}$ cell subsets count by flow cytometry is important in treatment followup. With the flow cytometry method, it is possible to examine all immune system subgroups and to conduct cytokine storm research. 5,6,7

Key-words: COVID-19, SARS-CoV-2, Rapid Antigen Testing, RT-PCR, Flow Cytometry 